\title{
MODULAÇÃO SPACE VECTOR PARA INVERSORES ALIMENTADOS EM TENSÃO: UMA ABORDAGEM UNIFICADA
}

\author{
Humberto Pinheiro* \\ humberto@ctlab.ufsm.br
}

\author{
Fernando Botterón* \\ botteron@gmail.com
}

\author{
Cassiano Rech* \\ cassiano@ieee.org
}

Luciano Schuch*

lschuch@mail.ufsm.br

\author{
Robinson F. Camargo* \\ robinson.camargo@gmail.com
}

\author{
Hélio L. Hey* \\ hey@smail.ufsm.br
}

\author{
Hilton A. Gründling* \\ ghilton@ctlab.ufsm.br
}

\author{
José R. Pinheiro* \\ renes@ctlab.ufsm.br
}

* Grupo de Eletrônica de Potência e Controle - Universidade Federal de Santa Maria

CEP 97105-900, Santa Maria, RS, BRASIL, +55-55-3220-8463

\begin{abstract}
Resumo: O objetivo deste trabalho é de que ele possa ser utilizado como tutorial da modulação space vector para profissionais da área de Eletrônica de Potência. Nesse sentido, este artigo apresenta uma abordagem unificada para a modulação space vector aplicada a inversores alimentados em tensão. Cinco inversores de tensão são apresentados para exemplificar a metodologia proposta, são eles: inversor monofásico em ponte completa, inversor trifásico a três fios de dois e três níveis, inversor trifásico a quatro fios de três e quatro braços. Para cada um dos inversores são apresentados os vetores de comutação, os planos de separação, os planos limites para operação na região linear, bem como algumas possíveis seqüências de comutação. Resultados experimentais são apresentados para validar a metodologia proposta.
\end{abstract}

Palavras Chaves: Modulação Space Vector, Técnicas de Modulação, Inversores de Tensão.

Abstract: This paper presents a unified approach of the space vector modulation for voltage-source inverters. To demonstrate the proposed unified approach, five fundamental inverters topologies are analyzed, that are: single-phase full-bridge; three-phase three-wire; threephase four-wire, three-phase four-leg and three-phase threelevel inverters. Switching vectors, separation and boundary planes in the inverter output space as well as decomposition

Artigo Submetido em 30/01/03

1a. Revisão em 21/09/04;

Aceito sob recomendação do Editor Associado

Prof. Dr. José Antenor Pomilio matrices and possible switching sequences are derived for each one of these inverters. Experimental results are shown to validate the proposed approach.

Keywords: Space Vector Modulation, Modulation Techniques, Voltage-source Inverters.

\section{INTRODUÇÃO}

Inversores alimentados em tensão são utilizados para produzir tensões alternadas em diversas aplicações, tais como fontes ininterruptas de energia (UPS's), acionamento de motores e filtros ativos. Assim, várias topologias de inversores têm sido desenvolvidas tanto para aplicações monofásicas quanto trifásicas. Diversas estratégias de modulação, diferentes em conceito e desempenho, têm sido desenvolvidas nas últimas décadas para a modulação destes inversores de tensão (Holtz, 1992). Dentre estas estratégias, pode-se citar a eliminação de harmônicas com modulação por largura de pulso (PWM) programada (Patel e Hoft, 1973; Enjeti et al., 1990). Esta técnica, possibilita eliminar de maneira seletiva as harmônicas de baixa ordem da tensão de saída de inversores. Entretanto, deve-se salientar que, para operação em malha fechada, deve-se limitar a banda passante do controlador de tensão para se obter o desempenho esperado. Outra estratégia de modulação aplicada em inversores é a modulação delta (DM), (Ziogas, 1981; Rahman et. Al., 1987). Esta estratégia apresenta boa atenuação de harmônicas de baixa ordem nas tensões de saída e reduzido número de comutações para índices de modulação de amplitude elevados. Entretanto, a freqüência de comutação resultante é variável. Além destas estratégias pode-se destacar a modulação por largura de pulso senoidal 
(SPWM). Esta apresenta freqüência de comutação constante e afasta as componentes harmônicas das tensões de saída do inversor da freqüência fundamental (Bowes e Lai, 1997; Boost e Ziogas, 1988). Na modulação SPWM para inversores trifásicos a três fios, o índice de modulação da amplitude da tensão de saída é limitado. Para contornar este problema, é possível injetar um sinal com terceira harmônica e seus múltiplos no sinal modulante (Boost e Ziogas, 1988). Uma abordagem geométrica da modulação por largura de pulso para inversores alimentados em tensão é apresentada por Ryan et al. (1999a e 1999b). Nesta abordagem, é definida uma transformação que relaciona o espaço das tensões de saída do inversor com o espaço das tensões produzidas pelos braços do inversor. Apesar de resultar em um sistema desacoplado, um problema adicional deve ser resolvido para a sua implementação, que é a definição da tensão de seqüência zero.

Por outro lado, as técnicas de modulação space vector têm sido muito aplicadas em inversores, pois permitem reduzir o número de comutações dos interruptores, diminuir o conteúdo harmônico da tensão de saída, e aumentar o índice de modulação de amplitude do inversor (Ranganathan, 1997; Van der Broeck, 1988; Wang, 2000). Além disso, a modulação space vector pode ser facilmente implementada em microprocessadores (Jenni e Wueest, 1993; Botterón et al., 2001; Celanovic e Boroyevich, 2001). A modulação space vector foi inicialmente aplicada em inversores trifásicos com três braços para acionameto de máquinas elétricas (Van der Broeck, 1988; Holtz, 1992). Posteriormente, foi também aplicada em conversores CACA trifásicos (Huber e Boroyevich, 1995; Casadei et. al., 2002) e monofásicos com três braços (Pinheiro et al., 2000), em inversores trifásicos com quatro braços (Zhang et. al., 1997) e em inversores trifásicos três níveis (Rojas et. al., 1995). Apesar da modulação space vector ter sido aplicada a diferentes conversores, até o momento não foi apresentada uma abordagem que saliente as características comuns desta estratégia. Sendo assim, este artigo desenvolve uma abordagem unificada da modulação do tipo space vector para inversores alimentados em tensão. Cinco inversores de tensão são apresentados para exemplificar a metodologia proposta. Para cada um dos inversores são mostrados os vetores de comutação, os planos de separação, os planos limites para operação na região linear, bem como algumas possíveis seqüências de comutação que podem ser implementadas, visando reduzir a taxa de distorção

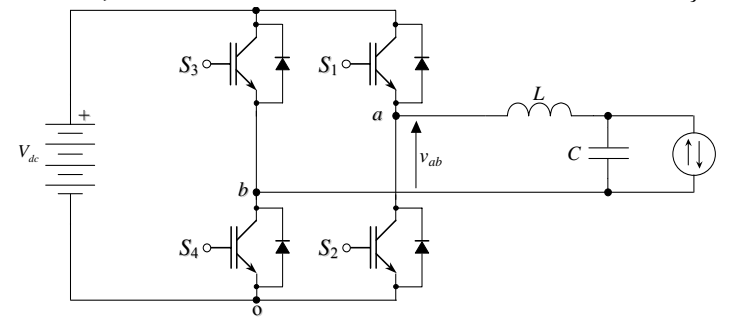

Figura 1. Inversor monofásico em ponte completa. harmônica (THD) da tensão de saída e/ou número de comutações dos interruptores. Resultados experimentais são apresentados para validar a metodologia proposta.

\section{GENERALIZAÇÃO DA MODULAÇÃO SPACE VECTOR}

Cinco etapas podem ser identificadas na implementação de uma modulação do tipo space vector para inversores de tensão (Camargo et al., 2002):

i. Definição dos possíveis vetores de comutação no espaço das tensões de saída do inversor.

ii. Identificação dos planos de separação dos setores no espaço das tensões de saída do inversor.

iii. Identificação dos planos limites no espaço das tensões de saída do inversor.

iv. Obtenção das matrizes de decomposição.

v. Definição da seqüência de comutação.

$\mathrm{Na}$ primeira etapa é possível incluir uma transformação no espaço das tensões de saída do inversor para simplificar a sua representação. Por outro lado, os planos de separação da segunda etapa podem ser utilizados no algoritmo de identificação do setor, onde o vetor das tensões de saída do inversor, a ser implementado, se encontra. Os planos limites, da terceira etapa, definem se um determinado vetor de tensão poderá ser implementado pelo inversor, e, em caso afirmativo, as matrizes de decomposição, da quarta etapa, possibilitam obter a duração de cada vetor de comutação em um período de comutação. Finalmente, as seqüências de comutação são escolhidas para minimizar uma grandeza adicional que pode incluir (Doval-Gandoy et al., 1999): (a) perdas em comutação; (b) taxa de distorção harmônica (THD); e em alguns inversores, (c) o desbalanceamento do ponto neutro do barramento CC. Nas próximas seções, esta metodologia é aplicada para diferentes inversores de tensão.

\section{INVERSOR MONOFÁSICO EM PONTE COMPLETA}

Nesta seção, os conceitos da modulação space vector, vistos na seção anterior, são aplicados ao inversor monofásico em ponte completa mostrado na Figura 1.

Assume-se que os interruptores $S_{1}$ e $S_{2}$ bem como $S_{3}$ e $S_{4}$ são comandados de forma complementar. Assim, existem 4 estados de condução do inversor, que são mostrados na Tabela 1, onde as tensões são normalizadas em função da tensão do barramento $\mathrm{CC}$, isto é: $v_{x}^{\prime}=v_{x} / V_{d c}$.

Uma vez que o inversor da Figura 1 produz apenas uma tensão, $v_{a b}$, que é aplicada à carga, esta pode ser representada sobre um espaço unidimensional, ou seja, 
sobre uma reta. Neste espaço unidimensional, os estados de condução do inversor definem 4 possíveis vetores de comutação, como mostrado na Figura 2. Dada uma tensão a ser produzida pelo inversor, aqui representada pelo vetor $\mathbf{u}_{c m d}$, existem diferentes combinações de vetores de comutação que podem ser usadas para sintetizar esta tensão. Porém, para minimizar as ondulações sobre as variáveis de interesse os vetores mais próximos de $\mathbf{u}_{c m d}$ devem ser escolhidos.

\subsection{Planos de Separação e Planos Limites}

Na Figura 2 pode-se definir dois setores distintos. Em cada um destes setores, o vetor de comando $\mathbf{u}_{c m d}$ compartilha três vetores de comutação mais próximos. Estes setores são: Setor 1 , onde $v_{a b}^{\prime}>0$ e, Setor 2, onde $v_{a b}^{\prime}<0$. Logo, neste caso existe apenas um "plano" de separação entre os setores, que é dado por:

$$
v_{a b}^{\prime}=0
$$

Por outro lado, os "planos" limites, que definem se a tensão $\mathbf{u}_{c m d}$ poderá ser sintetizada pelo inversor, são apresentados na Tabela 2. Por exemplo, no Setor 1, sempre que $\mathbf{u}_{c m d}>1$ pode-se definir uma tensão limitada a ser produzida pelo inversor da seguinte forma:

$$
\mathbf{u}_{c m d_{-} L}=\mathbf{u}_{c m d} /\left|\mathbf{u}_{c m d}\right|=1 \text {. }
$$

Tabela 2. Planos limites para o inversor monofásico em ponte completa.

\begin{tabular}{cc}
\hline Setor & "Plano" limite \\
\hline 1 & $v_{a b}^{\prime}=1$ \\
2 & $v_{a b}^{\prime}=-1$ \\
\hline
\end{tabular}

\subsection{Matrizes de Decomposição}

Uma vez identificado o setor em que $\mathbf{u}_{c m d}$ se localiza, devese obter a duração de cada vetor de comutação em um período de comutação $T_{s}$. Sem perda de generalidade, assume-se que a tensão a ser sintetizada pelo inversor está no Setor 1 , e que os vetores utilizados na implementação são $\mathbf{v}^{0}, \mathbf{v}^{1}$ e $\mathbf{v}^{3}$. Para que a tensão média produzida pelo inversor em $T_{s}$ seja igual a $\mathbf{u}_{c m d}$, a seguinte equação deve ser satisfeita:

$$
\mathbf{u}_{c m d}=\frac{1}{T_{s}}\left(\int_{0}^{t_{1}} \mathbf{v}^{0} d t+\int_{t_{1}}^{t_{2}} \mathbf{v}^{1} d t+\int_{t_{2}}^{T_{s}} \mathbf{v}^{3} d t\right) .
$$

Como $\mathbf{v}^{0}$ e $\mathbf{v}^{3}$ são vetores nulos, (3) pode ser reescrita como:

\begin{tabular}{|c|c|c|c|c|c|}
\hline$S_{1}$ & $S_{3}$ & $v_{a o}^{\prime}$ & $v_{b o}^{\prime}$ & $v_{a b}^{\prime}$ & Vetores \\
\hline 0 & 0 & 0 & 0 & 0 & $\mathbf{v}^{0}$ \\
\hline 1 & 0 & 1 & 0 & 1 & $\mathbf{v}^{1}$ \\
\hline 0 & 1 & 0 & 1 & -1 & $\mathbf{v}^{2}$ \\
\hline 1 & 1 & 1 & 1 & 0 & $\mathbf{v}^{3}$ \\
\hline & & $\mathbf{v}^{0}$ & & & \\
\hline 1$\}$ & & $6\}$ & & & \\
\hline
\end{tabular}

Tabela 1. Possíveis vetores de comutação para o inversor monofásico em ponte completa.

Figura 2. Espaço da tensão de saída para o inversor monofásico em ponte completa.

$$
\mathbf{u}_{c m d}=\frac{1}{T_{s}} \mathbf{v}^{1}\left(t_{2}-t_{1}\right)=\frac{1}{T_{s}} \mathbf{v}^{1} \Delta t_{1} .
$$

Uma vez que o vetor $\mathbf{v}^{1}$ é maior que zero, o tempo que o vetor $\mathbf{v}^{1}$ deve ser aplicado em $T_{s}$, pode ser obtido por,

$$
\Delta t_{1}=T_{s}\left(\mathbf{v}^{1}\right)^{-1} \mathbf{u}_{c m d}=T_{s} \mathbf{M}_{1} \mathbf{u}_{c m d},
$$

onde $\mathbf{M}_{1}$ é denominada matriz de decomposição, e o subscrito 1 é usado para indicar que essa matriz está associada ao Setor 1. Por outro lado, a duração dos vetores nulos, $\mathbf{v}^{0}$ e $\mathbf{v}^{3}$, são calculados da seguinte forma : $\Delta t_{0}+\Delta t_{3}$ $=T_{s}-\Delta t_{1}$. A definição específica de $\Delta t_{0}$ e $\Delta t_{3}$ bem como a distribuição dos vetores ao longo do período de comutação definem as diferentes seqüências de comutação.

\subsection{Seqüências de Comutação}

Note que, nas seções anteriores, os vetores de comutação, bem como as suas durações em um período de comutação $T_{s}$, foram definidos. A modulação space vector apresenta um grau de liberdade adicional, que é a escolha da distribuição dos vetores de comutação ao longo de $T_{s}$, o que resulta em diferentes seqüências de comutação. $\mathrm{Na}$ escolha de uma seqüência de comutação, é desejável assegurar que a transição entre diferentes vetores seja realizada pela comutação de somente um braço do inversor (Van der Broeck et. al., 1988; Ranganathan, 1997). Assim, haverá uma redução do número de comutações e, conseqüentemente, uma redução das perdas em comutação.

Várias seqüências de comutação podem ser geradas resultando em diferentes desempenhos. A Tabela 3 apresenta duas seqüências de comutação. A primeira, é chamada de simétrica, e é caracterizada por resultar em uma baixa THD na tensão de saída, e a segunda, por comutar um braço do inversor em baixa freqüência $(60 \mathrm{~Hz})$. 
Tabela 3. Seqüências de comutação para o inversor monofásico em ponte completa.

\begin{tabular}{ccc}
\hline Setor & Sequência simétrica & Um braço a $60 \mathrm{~Hz}$ \\
\hline 1 & $\mathbf{v}^{0}-\mathbf{v}^{1}-\mathbf{v}^{3}-\mathbf{v}^{1}-\mathbf{v}^{0}$ & $\mathbf{v}^{0}-\mathbf{v}^{1}$ \\
2 & $\mathbf{v}^{0}-\mathbf{v}^{2}-\mathbf{v}^{3}-\mathbf{v}^{2}-\mathbf{v}^{0}$ & $\mathbf{v}^{3}-\mathbf{v}^{2}$ \\
\hline
\end{tabular}

\section{INVERSOR TRIFÁSICO COM TRÊS BRAÇOS A TRÊS FIOS}

Nesta seção, serão aplicados os conceitos da modulação space vector, vistos na Seção 2, ao inversor trifásico três braços a três fios mostrado na Figura 3.

Novamente, assume-se que os interruptores $S_{1}$ e $S_{2}, S_{3}$ e $S_{4}$ bem como $S_{5}$ e $S_{6}$ são comandados de forma complementar, resultando em 8 possíveis estados de condução do inversor, mostrados na Tabela 4. Aplicando a lei de Kirchhoff das tensões na saída do inversor, conclui-se que $v_{a b}, v_{b c}$, e $v_{c a}$, são linearmente dependentes. Como resultado, os possíveis vetores de comutação, estão sobre um plano no sistema de coordenadas definido por essas tensões, como mostrado na Figura 4. Portanto, esses vetores podem ser representados em um espaço bidimensional. Com o objetivo de simplificar a representação dos possíveis vetores de comutação, pode-se utilizar uma transformação linear de $\mathfrak{R}^{3} \rightarrow \mathfrak{R}^{2}$. Geralmente, é utilizada a transformação

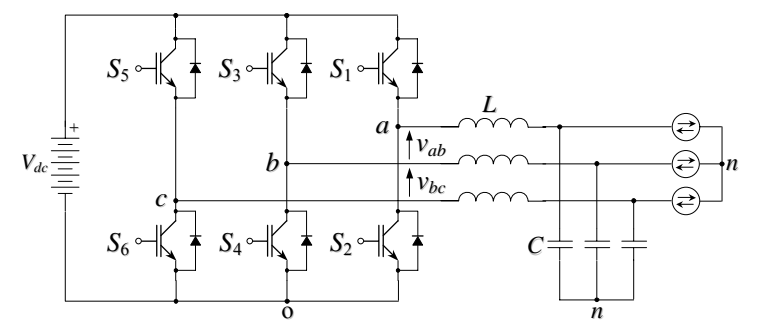

Figura 3. Inversor trifásico com três braços a três fios.

Tabela 4. Possíveis vetores de comutação para o inversor trifásico com três braços a três fios.

\begin{tabular}{cccccccccc}
\hline$S_{1}$ & $S_{3}$ & $S_{5}$ & $v_{a o}^{\prime}$ & $v_{b o}^{\prime}$ & $v_{c o}^{\prime}$ & $v_{a b}^{\prime}$ & $v_{b c}^{\prime}$ & $v_{c a}^{\prime}$ & Vetores \\
\hline 0 & 0 & 0 & 0 & 0 & 0 & 0 & 0 & 0 & $\mathbf{v}^{0}$ \\
0 & 0 & 1 & 0 & 0 & 1 & 0 & -1 & 1 & $\mathbf{v}^{1}$ \\
0 & 1 & 0 & 0 & 1 & 0 & -1 & 1 & 0 & $\mathbf{v}^{2}$ \\
0 & 1 & 1 & 0 & 1 & 1 & -1 & 0 & 1 & $\mathbf{v}^{3}$ \\
1 & 0 & 0 & 1 & 0 & 0 & 1 & 0 & -1 & $\mathbf{v}^{4}$ \\
1 & 0 & 1 & 1 & 0 & 1 & 1 & -1 & 0 & $\mathbf{v}^{5}$ \\
1 & 1 & 0 & 1 & 1 & 0 & 0 & 1 & -1 & $\mathbf{v}^{6}$ \\
1 & 1 & 1 & 1 & 1 & 1 & 0 & 0 & 0 & $\mathbf{v}^{7}$ \\
\hline
\end{tabular}

denominada $\alpha \beta$ (Ranganathan, 1997), que é:

$$
\mathbf{T}_{\alpha \beta}=\sqrt{\frac{2}{3}}\left[\begin{array}{ccc}
1 & -\frac{1}{2} & -\frac{1}{2} \\
0 & \frac{\sqrt{3}}{2} & -\frac{\sqrt{3}}{2}
\end{array}\right] .
$$

Aplicando-se a transformação (6) aos oito possíveis vetores de comutação definidos na Tabela 4, obtém-se uma projeção ortogonal do espaço das tensões em $[a b c]^{\mathrm{T}}$, em um plano perpendicular ao vetor $\left[\begin{array}{lll}1 & 1 & 1\end{array}\right]^{\mathrm{T}}$. Neste novo sistema de coordenadas, $\alpha \beta$, tem-se: 6 vetores não nulos (cujos extremos definem os vertices de um hexágono regular), que possuem um ângulo de $60^{\circ}$ entre eles, com módulo igual a $\sqrt{2 / 3}$, e dois vetores com módulo zero, como mostrado na Figura 5.

\subsection{Planos de Separação e Planos Limites}

No espaço das tensões de saída mostrado na Figura 5, podem

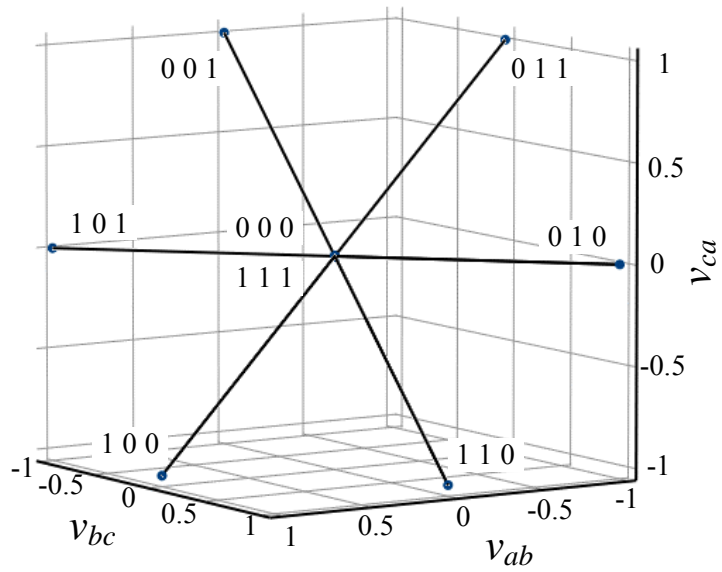

Figura 4. Espaço das tensões de saída no sistema de coordenas abc para o inversor trifásico com três braços a três fios.

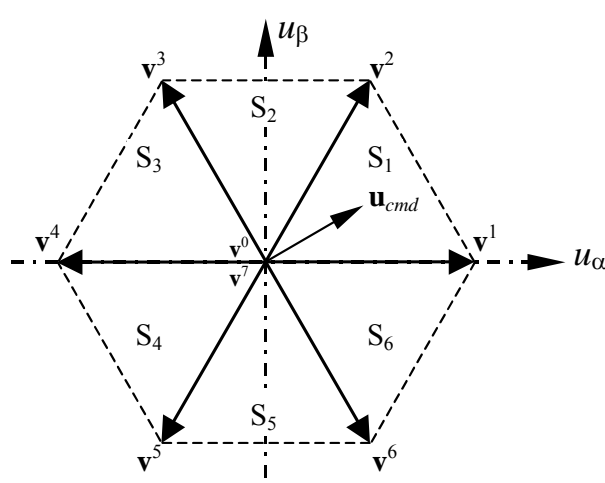

Figura 5. Espaço das tensões de saída no sistema de coordenadas $\alpha \beta$ para o inversor trifásico com três braços a três fios. 
ser definidos seis setores distintos. Em cada um destes setores, existem dois vetores de comutação não nulos adjacentes ao vetor de comando $\mathbf{u}_{c m d}$. Os "planos" de separação dos setores em coordenadas $\alpha \beta$ são definidos pelas seguintes equações:

$$
\begin{gathered}
\mathrm{PS}_{1} \therefore u_{\beta}+\sqrt{3} u_{\alpha}=0, \mathrm{PS}_{2} \therefore u_{\beta}-\sqrt{3} u_{\alpha}=0, \\
\mathrm{PS}_{3} \therefore u_{\beta}=0
\end{gathered}
$$

Por outro lado, a partir dos vértices dos possíveis vetores de comutação define-se o "plano" limite de cada setor. Estes são apresentados na Tabela 5.

Tabela 5. Planos limites para o inversor trifásico com três braços a três fios.

\begin{tabular}{cc}
\hline Setor & Plano Limite \\
\hline 1 & $\mathrm{PL}_{1} \therefore u_{\beta}+\sqrt{3} u_{\alpha}-\sqrt{2}=0$ \\
2 & $\mathrm{PL}_{2} \therefore u_{\beta}-\sqrt{2} / 2=0$ \\
3 & $\mathrm{PL}_{3} \therefore u_{\beta}-\sqrt{3} u_{\alpha}-\sqrt{2}=0$ \\
4 & $\mathrm{PL}_{4} \therefore u_{\beta}+\sqrt{3} u_{\alpha}+\sqrt{2}=0$ \\
5 & $\mathrm{PL}_{5} \therefore u_{\beta}+\sqrt{2} / 2=0$ \\
6 & $\mathrm{PL}_{6} \therefore u_{\beta}-\sqrt{3} u_{\alpha}+\sqrt{2}=0$ \\
\hline
\end{tabular}

\subsection{Matrizes de Decomposição}

Após identificar em qual setor se localiza o vetor $\mathbf{u}_{c m d}$, deve-se obter a duração de cada vetor de comutação em um período de comutação $T_{s}$. Sem perda de generalidade, assume-se que o vetor de comando $\mathbf{u}_{c m d}$ está no Setor 1 , e que a seqüência de comutação implementada é $\mathbf{v}^{0}, \mathbf{v}^{1}, \mathbf{v}^{2}, \mathbf{v}^{7}, \mathbf{v}^{2}, \mathbf{v}^{1}, \mathbf{v}^{0}$. A duração total de aplicação de cada vetor de comutação $\left(\mathbf{v}^{1}, \mathbf{v}^{2}, \mathbf{v}^{0} / \mathbf{v}^{7}\right) \mathrm{em}$ um período $T_{s}$ foram denominados por: $\Delta t_{1}, \Delta t_{2}$ e $\Delta t_{0}$, respectivamente. Então, para que a tensão média sintetizada pelo inversor em um período $T_{s}$ seja igual a $\mathbf{u}_{c m d}$, a equação (8) deve ser satisfeita:

$$
\mathbf{v}^{1} \Delta t_{1}+\mathbf{v}^{2} \Delta t_{2}+\left(\mathbf{v}^{0} \text { ou } \mathbf{v}^{7}\right) \Delta t_{0}=\mathbf{u}_{c m d} T_{s}
$$

onde: $\Delta t_{1}+\Delta t_{2}+\Delta t_{0}=T_{s}$.

Uma vez que $\mathbf{v}^{0}$ e $\mathbf{v}^{7}$ são vetores nulos, a equação (8) pode ser reescrita na seguinte forma matricial:

$$
\left[\begin{array}{ll}
\mathbf{v}^{1} & \mathbf{v}^{2}
\end{array}\right]\left[\begin{array}{ll}
\Delta t_{1} & \Delta t_{2}
\end{array}\right]^{\mathrm{T}}=\mathbf{u}_{c m d} T_{s} .
$$

Então, como os vetores $\mathbf{v}^{1}$ e $\mathbf{v}^{2}$ são linearmente independentes, as durações $\Delta t_{1}$ e $\Delta t_{2}$ podem ser obtidas por:

$$
\left[\begin{array}{c}
\Delta t_{1} \\
\Delta t_{2}
\end{array}\right]=\mathbf{M}_{1}\left[\begin{array}{l}
u_{\alpha} \\
u_{\beta}
\end{array}\right] T_{s} \quad \therefore \quad \mathbf{M}_{1}=\left[\begin{array}{ll}
\mathbf{v}^{1} & \mathbf{v}^{2}
\end{array}\right]^{-1}
$$

onde $u_{\alpha}$ e $u_{\beta}$ são as componentes do vetor de comando $\mathbf{u}_{c m d}$, e $\mathbf{M}_{1}$ é a matriz de decomposição associada ao Setor 1. Por outro lado, a duração dos vetores nulos são calculados a partir da seguinte forma: $\Delta t_{0}=T_{s}-\Delta t_{1}-\Delta t_{2}$.

\subsection{Seqüências de Comutação}

Com base nas considerações efetuadas na seção anterior, a Tabela 6 apresenta duas possíveis seqüências de comutação. Na primeira, chamada simétrica, os três braços do inversor comutam em alta freqüência, e, devido à simetria do padrão PWM gerado, resulta em uma baixa THD nas tensões de saída. A segunda caracteriza-se por, em cada setor, manter um braço diferente do inversor sem comutar.

Tabela 6. Seqüências de comutação para o inversor trifásico com três braços a três fios.

\begin{tabular}{ccc}
\hline Setor & Primeira Seqüência & Segunda Seqüência \\
\hline 1 & $\mathbf{v}^{0}-\mathbf{v}^{1}-\mathbf{v}^{2}-\mathbf{v}^{7}-\mathbf{v}^{2}-\mathbf{v}^{1}-\mathbf{v}^{0}$ & $\mathbf{v}^{1}-\mathbf{v}^{2}-\mathbf{v}^{7}-\mathbf{v}^{2}-\mathbf{v}^{1}$ \\
2 & $\mathbf{v}^{0}-\mathbf{v}^{3}-\mathbf{v}^{2}-\mathbf{v}^{7}-\mathbf{v}^{2}-\mathbf{v}^{3}-\mathbf{v}^{0}$ & $\mathbf{v}^{2}-\mathbf{v}^{3}-\mathbf{v}^{0}-\mathbf{v}^{3}-\mathbf{v}^{2}$ \\
3 & $\mathbf{v}^{0}-\mathbf{v}^{3}-\mathbf{v}^{4}-\mathbf{v}^{7}-\mathbf{v}^{4}-\mathbf{v}^{3}-\mathbf{v}^{0}$ & $\mathbf{v}^{3}-\mathbf{v}^{4}-\mathbf{v}^{7}-\mathbf{v}^{4}-\mathbf{v}^{3}$ \\
4 & $\mathbf{v}^{0}-\mathbf{v}^{5}-\mathbf{v}^{4}-\mathbf{v}^{7}-\mathbf{v}^{4}-\mathbf{v}^{5}-\mathbf{v}^{0}$ & $\mathbf{v}^{4}-\mathbf{v}^{5}-\mathbf{v}^{0}-\mathbf{v}^{5}-\mathbf{v}^{4}$ \\
5 & $\mathbf{v}^{0}-\mathbf{v}^{5}-\mathbf{v}^{6}-\mathbf{v}^{7}-\mathbf{v}^{6}-\mathbf{v}^{5}-\mathbf{v}^{0}$ & $\mathbf{v}^{5}-\mathbf{v}^{6}-\mathbf{v}^{7}-\mathbf{v}^{6}-\mathbf{v}^{5}$ \\
6 & $\mathbf{v}^{0}-\mathbf{v}^{1}-\mathbf{v}^{6}-\mathbf{v}^{7}-\mathbf{v}^{6}-\mathbf{v}^{1}-\mathbf{v}^{0}$ & $\mathbf{v}^{6}-\mathbf{v}^{1}-\mathbf{v}^{0}-\mathbf{v}^{1}-\mathbf{v}^{6}$ \\
\hline
\end{tabular}

\section{INVERSOR TRIFÁSICO COM TRÊS BRAÇOS A QUATRO FIOS}

Na Figura 6 é apresentado o inversor trifásico com três braços a quatro fios. De forma semelhante ao inversor trifásico com três braços a três fios, neste caso também existem 8 possíveis vetores de comutação (Zhang et al., 1997; Zhan et al., 2001).

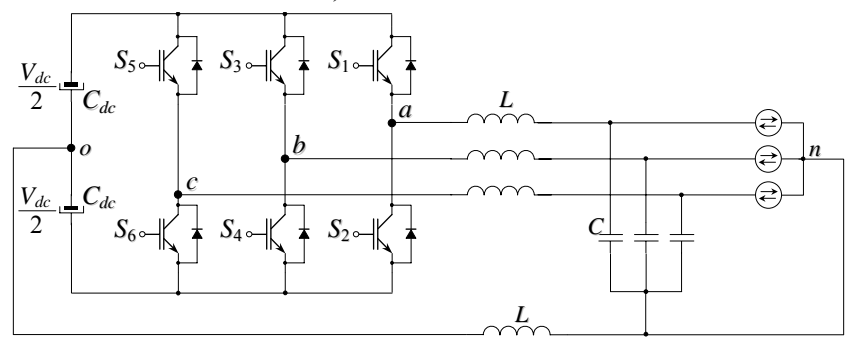

Figura 6. Inversor trifásico com três braços a quatro fios.

As possíveis tensões de saída do inversor em relação ao ponto central do barramento $\mathrm{CC}$ são apresentadas na Tabela 7. Essas tensões são normalizadas com relação a $V_{d c}$, considerando que as tensões nos capacitores do barramento CC são iguais, ou seja, $V_{d c 1}=V_{d c 2}=V_{d c} / 2$. 


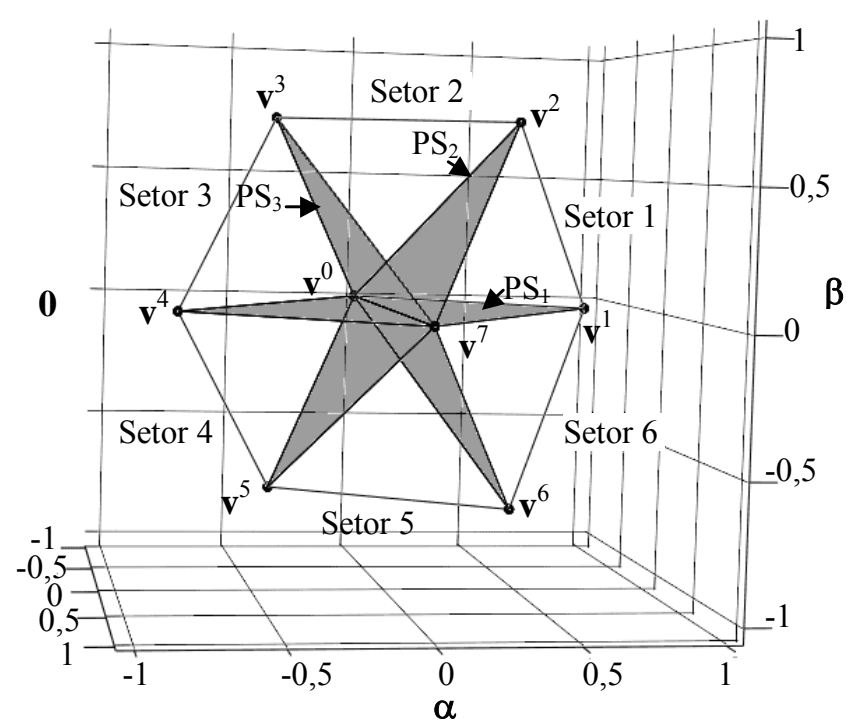

Figura 7. Espaço das tensões de saída do inversor trifásico com três braços a quatro fios em coordenadas $0 \alpha \beta$.

Tabela 7. Possíveis vetores de comutação do inversor trifásico com três braços a quatro fios.

\begin{tabular}{ccccccccc}
\hline$S_{1}$ & $S_{3}$ & $S_{5}$ & $v_{a o}^{\prime}$ & $v_{b o}^{\prime}$ & $v_{c o}^{\prime}$ & {$\left[\begin{array}{lll}0 & \alpha & \beta\end{array}\right]^{\mathrm{T}}$} & Vetores \\
\hline 0 & 0 & 0 & $-1 / 2$ & $-1 / 2$ & $-1 / 2$ & {$\left[\begin{array}{lllll}-\sqrt{3} / 2 & 0 & 0\end{array}\right]$} & $\mathbf{v}^{0}$ \\
1 & 0 & 0 & $1 / 2$ & $-1 / 2$ & $-1 / 2$ & {$\left[\begin{array}{lllll}-\sqrt{3} / 6 & \sqrt{6} / 3 & 0\end{array}\right]$} & $\mathbf{v}^{1}$ \\
1 & 1 & 0 & $1 / 2$ & $1 / 2$ & $-1 / 2$ & {$\left[\begin{array}{lllll}\sqrt{3} / 6 & 1 / \sqrt{6} & \sqrt{2} / 2\end{array}\right]$} & $\mathbf{v}^{2}$ \\
0 & 1 & 0 & $-1 / 2$ & $1 / 2$ & $-1 / 2$ & {$\left[\begin{array}{lllll}-\sqrt{3} / 6 & -1 / \sqrt{6} & \sqrt{2} / 2\end{array}\right]$} & $\mathbf{v}^{3}$ \\
0 & 1 & 1 & $-1 / 2$ & $1 / 2$ & $1 / 2$ & {$\left[\begin{array}{ccccc}\sqrt{3} / 6 & -\sqrt{6} / 3 & 0\end{array}\right]$} & $\mathbf{v}^{4}$ \\
0 & 0 & 1 & $-1 / 2$ & $-1 / 2$ & $1 / 2$ & {$\left[\begin{array}{llll}-\sqrt{3} / 6 & -1 / \sqrt{6} & -\sqrt{2} / 2\end{array}\right]$} & $\mathbf{v}^{5}$ \\
1 & 0 & 1 & $1 / 2$ & $-1 / 2$ & $1 / 2$ & {$\left[\begin{array}{cccc}\sqrt{3} / 6 & 1 / \sqrt{6} & -\sqrt{2} / 2\end{array}\right]$} & $\mathbf{v}^{6}$ \\
1 & 1 & 1 & $1 / 2$ & $1 / 2$ & $1 / 2$ & {$\left[\begin{array}{ccccc}\sqrt{3} / 2 & 0 & 0\end{array}\right]$} & $\mathbf{v}^{7}$ \\
\hline
\end{tabular}

Neste caso, o inversor sintetiza três tensões de saída linearmente independentes, que podem ser representadas em um espaço tridimensional. Aqui utilizou-se a transformação $\mathfrak{R}^{3} \rightarrow \mathfrak{R}^{3}$, ortonormal $0 \alpha \beta$ definida por:

$$
T_{0 \alpha \beta}=\sqrt{\frac{2}{3}}\left[\begin{array}{ccc}
1 / \sqrt{2} & 1 / \sqrt{2} & 1 / \sqrt{2} \\
1 & -1 / 2 & -1 / 2 \\
0 & \sqrt{3} / 2 & -\sqrt{3} / 2
\end{array}\right],
$$

Com essa transformação, pode-se obter os vetores de comutação no espaço $0 \alpha \beta$, como mostrados na Tabela 7 .

\subsection{Planos de Separação e Planos Limites}

A Figura 7, mostra que os vetores de comutação, definem um poliedro que pode ser dividido em seis setores (tetraedros). Se o vetor de comando $\mathbf{u}_{c m d}$ estiver localizado em um desses setores, pode-se usar os quatro vetores associados a este tetraedro para sintetizar o vetor de comando em questão. Da Figura 7 verifica-se a existência de três planos de separação $\left(\mathrm{PS}_{1}, \mathrm{PS}_{2}\right.$ e $\left.\mathrm{PS}_{3}\right)$, que podem ser utilizados no algoritmo de identificação do setor. Estes planos de separação são definidos pelas seguintes equações:

$$
\begin{gathered}
\mathrm{PS}_{1} \therefore \frac{\sqrt{2}}{2} u_{\beta}=0, \mathrm{PS}_{2} \therefore \frac{-\sqrt{6}}{4} u_{\alpha}+\frac{\sqrt{2}}{4} u_{\beta}=0 \mathrm{e} \\
\mathrm{PS}_{3} \therefore \frac{-\sqrt{6}}{4} u_{\alpha}-\frac{\sqrt{2}}{4} u_{\beta}=0
\end{gathered}
$$

$\mathrm{Na}$ mesma figura pode-se identificar os planos limites que determinam a região de operação linear do inversor trifásico com três braços a quatro fios. Estes planos podem ser representados por:

$$
\begin{aligned}
& \mathrm{PL}_{1} \therefore-\frac{\sqrt{3}}{3} u_{o}+\sqrt{\frac{2}{3}} u_{\alpha}+\frac{\sqrt{2}}{2} u_{\beta}=0, \mathrm{PL}_{2} \therefore \frac{\sqrt{3}}{3} u_{o}+\sqrt{\frac{8}{3}} u_{\alpha}=0, \\
& \mathrm{PL}_{3} \therefore \frac{\sqrt{3}}{3} u_{o}-\sqrt{\frac{2}{3}} u_{\alpha}+\frac{\sqrt{2}}{2} u_{\beta}=0, \mathrm{PL}_{4} \therefore-\frac{\sqrt{3}}{3} u_{o}-\sqrt{\frac{8}{3}} u_{\alpha}=0, \\
& \mathrm{PL}_{5} \therefore \frac{\sqrt{3}}{3} u_{o}-\sqrt{\frac{2}{3}} u_{\alpha}-\frac{\sqrt{2}}{2} u_{\beta}=0, \\
& \mathrm{PL}_{6} \therefore-\frac{\sqrt{3}}{3} u_{o}+\sqrt{\frac{2}{3}} u_{\alpha}-\frac{\sqrt{2}}{2} u_{\beta}=0
\end{aligned}
$$

Cada setor é delimitado por dois planos limites, isto é: Setor 1, $\mathrm{PL}_{1}$ e $\mathrm{PL}_{2}$. Setor 2, $\mathrm{PL}_{1}$ e $\mathrm{PL}_{3}$. Setor 3, $\mathrm{PL}_{4}$ e $\mathrm{PL}_{3}$. Setor 4, $\mathrm{PL}_{4}$ e $\mathrm{PL}_{5}$. Setor 5, $\mathrm{PL}_{6}$ e $\mathrm{PL}_{5}$. Setor 6, $\mathrm{PL}_{6}$ e $\mathrm{PL}_{2}$.

\subsection{Matrizes de Decomposição}

Após identificar o tetraedro em que o vetor de comando se localiza, deve-se calcular a duração de cada vetor de comutação adjacente a $\mathbf{u}_{c m}$, em um período $T_{s}$. Novamente, assume-se que $\mathbf{u}_{c m d}$ está no Setor 1 , e que os vetores utilizados na implementação são $\mathbf{v}^{0}, \mathbf{v}^{7}, \mathbf{v}^{1}$ e $\mathbf{v}^{2}$. Então, para que a tensão média produzida pelo inversor em um período de comutação seja igual a $\mathbf{u}_{c m d}$ a seguinte equação deve ser satisfeita:

$$
\mathbf{v}^{0} \Delta t_{0}+\mathbf{v}^{7} \Delta t_{7}+\mathbf{v}^{1} \Delta t_{1}+\mathbf{v}^{2} \Delta t_{2}=\mathbf{u}_{c m d} T_{s},
$$

onde $\Delta t_{0}+\Delta t_{7}+\Delta t_{1}+\Delta t_{2}=T_{s}$.

Rescrevendo (13) na forma matricial obtém-se: 


$$
\left[\begin{array}{cccc}
\mathbf{v}^{0} & \mathbf{v}^{7} & \mathbf{v}^{1} & \mathbf{v}^{2} \\
1 & 1 & 1 & 1
\end{array}\right]\left[\begin{array}{c}
\Delta t_{0} \\
\Delta t_{7} \\
\Delta t_{1} \\
\Delta t_{2}
\end{array}\right]=\left[\begin{array}{c}
\mathbf{u}_{c m d} \\
1
\end{array}\right] T_{s}
$$

A última linha adicionada à matriz de vetores de comutação associada ao setor 1, faz com que essa matriz possua inversa. Assim, as durações podem ser determinados da seguinte forma:

$$
\left[\begin{array}{llll}
\Delta t_{0} & \Delta t_{7} & \Delta t_{1} & \Delta t_{2}
\end{array}\right]^{\mathrm{T}}=\mathbf{M}_{1}\left[\begin{array}{llll}
u_{0} & u_{\alpha} & u_{\beta} & 1
\end{array}\right]^{\mathrm{T}} T_{s}
$$

onde $u_{0}, u_{\alpha}$ e $u_{\beta}$ são as componentes do vetor de comando $\mathbf{u}_{c m d}, \Delta t_{0}, \Delta t_{7}, \Delta t_{1}$ e $\Delta t_{2}$ são as correspondentes durações de cada vetor e $\mathbf{M}_{1}$ é a matriz de decomposição associada ao Setor 1 .

\subsection{Seqüências de Comutação}

De forma similar ao apresentado nas seções anteriores, uma possível seqüência de comutação simétrica, que minimiza a THD da tensão de saída, é mostrada na Tabela 8.

Tabela 8. Seqüência de comutação simétrica para o inversor trifásico com três braços a quatro fios.

\begin{tabular}{ll|ll}
\hline Setor 1 & $\mathbf{v}^{0}-\mathbf{v}^{1}-\mathbf{v}^{2}-\mathbf{v}^{7}-\mathbf{v}^{2}-\mathbf{v}^{1}-\mathbf{v}^{0}$ & Setor 2 & $\mathbf{v}^{0}-\mathbf{v}^{3}-\mathbf{v}^{2}-\mathbf{v}^{7}-\mathbf{v}^{2}-\mathbf{v}^{3}-\mathbf{v}^{0}$ \\
\hline Setor 3 & $\mathbf{v}^{0}-\mathbf{v}^{3}-\mathbf{v}^{4}-\mathbf{v}^{7}-\mathbf{v}^{4}-\mathbf{v}^{3}-\mathbf{v}^{0}$ & Setor 4 & $\mathbf{v}^{0}-\mathbf{v}^{5}-\mathbf{v}^{4}-\mathbf{v}^{7}-\mathbf{v}^{4}-\mathbf{v}^{5}-\mathbf{v}^{0}$ \\
\hline Setor 5 & $\mathbf{v}^{0}-\mathbf{v}^{5}-\mathbf{v}^{6}-\mathbf{v}^{7}-\mathbf{v}^{6}-\mathbf{v}^{5}-\mathbf{v}^{0}$ & Setor 6 & $\mathbf{v}^{0}-\mathbf{v}^{1}-\mathbf{v}^{6}-\mathbf{v}^{7}-\mathbf{v}^{6}-\mathbf{v}^{1}-\mathbf{v}^{0}$ \\
\hline
\end{tabular}

\section{INVERSOR TRIFÁSICO COM QUATRO BRAÇOS A QUATRO FIOS}

Na Figura 8 é apresentado o inversor trifásico com quatro braços a quatros fios.

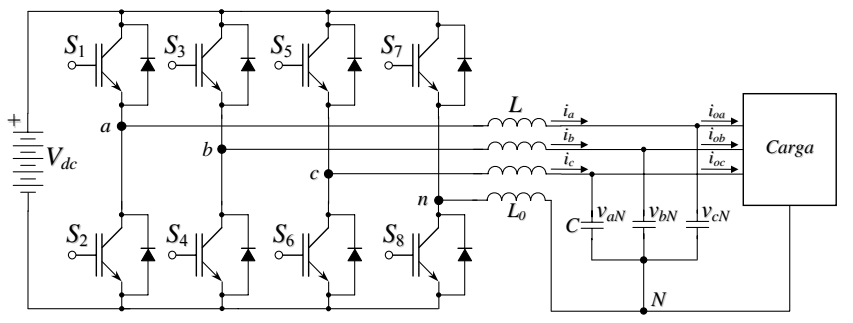

Figura 8. Inversor trifásico com quatro braços a quatro fios

Novamente, assume-se que os interruptores $S_{1}$ e $S_{2}, S_{3}$ e $S_{4}$, $S_{5}$ e $S_{6}$, bem como $S_{7}$ e $S_{8}$ são comandados de forma complementar. Logo, existem 16 possíveis estados de condução, que são apresentados na Tabela 9. Este inversor sintetiza três tensões de saída linearmente independentes, que podem ser representadas em um espaço tridimensional.
Neste espaço tridimensional, os estados de condução do inversor definem 16 possíveis vetores de comutação.

Tabela 9. Possíveis vetores de comutação para o inversor trifásico com quatro braços a quatro fios e tensões de fase em coordenadas abc e $0 \alpha \beta$.

\begin{tabular}{ccccccccccc}
\hline Vetores & $S_{a}$ & $S_{b}$ & $S_{c}$ & $S_{n}$ & $v_{a n}^{\prime}$ & $v_{b n}^{\prime}$ & $v_{c n}^{\prime}$ & $v_{0}$ & $v_{\alpha}$ & $v_{\beta}$ \\
\hline $\mathbf{v}^{0}$ & 0 & 0 & 0 & 0 & 0 & 0 & 0 & 0 & 0 & 0 \\
$\mathbf{v}^{1}$ & 0 & 0 & 0 & 1 & -1 & -1 & -1 & $-\sqrt{3}$ & 0 & 0 \\
$\mathbf{v}^{2}$ & 0 & 0 & 1 & 0 & 0 & 0 & 1 & $\sqrt{3} / 3$ & $-\sqrt{6} / 6$ & $-\sqrt{2} / 2$ \\
$\mathbf{v}^{3}$ & 0 & 0 & 1 & 1 & -1 & -1 & 0 & $-2 \sqrt{3} / 3$ & $-\sqrt{6} / 6$ & $-\sqrt{2} / 2$ \\
$\mathbf{v}^{4}$ & 0 & 1 & 0 & 0 & 0 & 1 & 0 & $\sqrt{3} / 3$ & $-\sqrt{6} / 6$ & $\sqrt{2} / 2$ \\
$\mathbf{v}^{5}$ & 0 & 1 & 0 & 1 & -1 & 0 & -1 & $-2 \sqrt{3} / 3$ & $-\sqrt{6} / 6$ & $\sqrt{2} / 2$ \\
$\mathbf{v}^{6}$ & 0 & 1 & 1 & 0 & 0 & 1 & 1 & $2 \sqrt{3} / 3$ & $-\sqrt{6} / 3$ & 0 \\
$\mathbf{v}^{7}$ & 0 & 1 & 1 & 1 & -1 & 0 & 0 & $-\sqrt{3} / 3$ & $-\sqrt{6} / 3$ & 0 \\
$\mathbf{v}^{8}$ & 1 & 0 & 0 & 0 & 1 & 0 & 0 & $\sqrt{3} / 3$ & $\sqrt{6} / 3$ & 0 \\
$\mathbf{v}^{9}$ & 1 & 0 & 0 & 1 & 0 & -1 & -1 & $-2 \sqrt{3} / 3$ & $\sqrt{6} / 3$ & 0 \\
$\mathbf{v}^{10}$ & 1 & 0 & 1 & 0 & 1 & 0 & 1 & $2 \sqrt{3} / 3$ & $\sqrt{6} / 6$ & $-\sqrt{2} / 2$ \\
$\mathbf{v}^{11}$ & 1 & 0 & 1 & 1 & 0 & -1 & 0 & $-\sqrt{3} / 3$ & $\sqrt{6} / 6$ & $-\sqrt{2} / 2$ \\
$\mathbf{v}^{12}$ & 1 & 1 & 0 & 0 & 1 & 1 & 0 & $2 \sqrt{3} / 3$ & $\sqrt{6} / 6$ & $\sqrt{2} / 2$ \\
$\mathbf{v}^{13}$ & 1 & 1 & 0 & 1 & 0 & 0 & -1 & $\sqrt{3} / 3$ & $\sqrt{6} / 6$ & $\sqrt{2} / 2$ \\
$\mathbf{v}^{14}$ & 1 & 1 & 1 & 0 & 1 & 1 & 1 & $\sqrt{3}$ & 0 & 0 \\
$\mathbf{v}^{15}$ & 1 & 1 & 1 & 1 & 0 & 0 & 0 & 0 & 0 & 0 \\
\hline & & & & & & & & & \\
\hline
\end{tabular}

Logo, utilizando a transformação (11), pode-se representar os possíveis vetores de comutação no espaço $0 \alpha \beta$, como mostrado na Figura 9(a). Os vértices destes 16 vetores de comutação definem um dodecaedro conforme mostra a Figura 9(b).

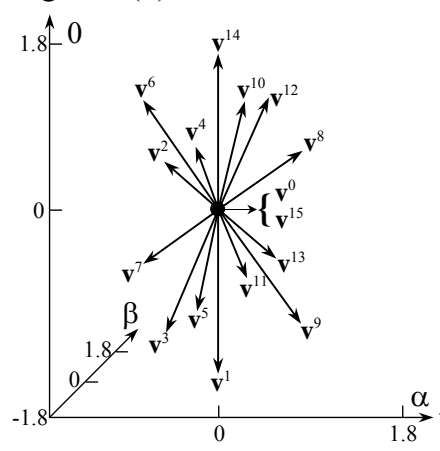

(a)

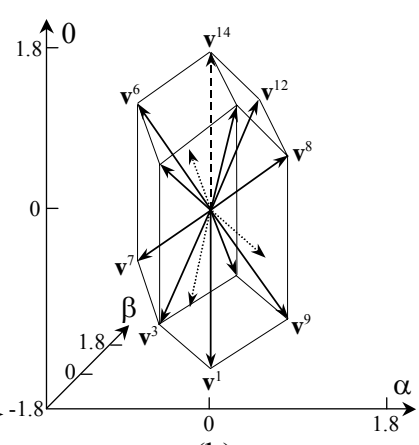

(b)
Figura 9. Inversor trifásico com quatro braços a quatro fios.(a) Espaço das tensões de saída. (b) Dodecaedro formado pelos vértices dos vetores de comutação. 


\subsection{Planos de Separação e Planos Limites}

No espaço das tensões de saída mostrado na Figura 9(a) podem ser definidos 24 setores (tetraedros) distintos. Em cada um destes setores, existem três vetores de comutação não nulos adjacentes ao vetor de comando $\mathbf{u}_{c m d}$. Os planos de separação dos setores em coordenadas $0 \alpha \beta$ podem ser definidos pelas seguintes equações:

$$
\begin{aligned}
& \mathrm{PS}_{1} \therefore \sqrt{2} u_{\beta}=0 \\
& \mathrm{PS}_{2} \therefore-\sqrt{6} / 2 u_{\alpha}+\sqrt{2} / 2 u_{\beta}=0 \\
& \mathrm{PS}_{3} \therefore \sqrt{6} / 2 u_{\alpha}+\sqrt{2} / 2 u_{\beta}=0 \\
& \mathrm{PS}_{4} \therefore \sqrt{3} / 3 u_{0}+\sqrt{6} / 3 u_{\alpha}=0 \\
& \mathrm{PS}_{5} \therefore \sqrt{3} / 3 u_{0}-\sqrt{6} / 6 u_{\alpha}-\sqrt{2} / 2 u_{\beta}=0 \\
& \mathrm{PS}_{6} \therefore \sqrt{3} / 3 u_{0}-\sqrt{6} / 6 u_{\alpha}+\sqrt{2} / 2 u_{\beta}=0
\end{aligned}
$$

Por outro lado, os vértices dos vetores de comutação não nulos definem 12 planos limites do dodecaedro, que determinam a região de operação linear do inversor trifásico com quatro braços a quatro fios. Estes planos podem ser representados em coordenadas $0 \alpha \beta$ por:

$$
\begin{aligned}
& \mathrm{PL}_{1} \therefore \sqrt{3} / 3 u_{0}+\sqrt{3} / 2 u_{\alpha}=1 \\
& \mathrm{PL}_{2} \therefore \sqrt{3} / 3 u_{0}-\sqrt{6} / 6 u_{\alpha}+\sqrt{2} / 2 u_{\beta}=1 \\
& \mathrm{PL}_{3} \therefore \sqrt{3} / 3 u_{0}-\sqrt{6} / 6 u_{\alpha}-\sqrt{2} / 2 u_{\beta}=1 \\
& \mathrm{PL}_{4} \therefore \sqrt{6} / 2 u_{\alpha}+\sqrt{2} / 2 u_{\beta}=1 \\
& \mathrm{PL}_{5} \therefore \sqrt{2} u_{\beta}=1 \\
& \mathrm{PL}_{6} \therefore-\sqrt{6} / 2 u_{\alpha}+\sqrt{2} / 2 u_{\beta}=1 \\
& \mathrm{PL}_{7} \therefore-\sqrt{6} / 2 u_{\alpha}-\sqrt{2} / 2 u_{\beta}=1 \\
& \mathrm{PL}_{8} \therefore-\sqrt{2} u_{\beta}=1 \\
& \mathrm{PL}_{9} \therefore \sqrt{6} / 2 u_{\alpha}-\sqrt{2} / 2 u_{\beta}=1 \\
& \mathrm{PL}_{10} \therefore-\sqrt{3} / 3 u_{0}+\sqrt{6} / 6 u_{\alpha}+\sqrt{2} / 2 u_{\beta}=1 \\
& \mathrm{PL}_{11} \therefore-\sqrt{3} / 3 u_{0}-\sqrt{2 / 3} u_{\alpha}=1 \\
& \mathrm{PL}_{12} \therefore-\sqrt{3} / 3 u_{0}+\sqrt{6} / 6 u_{\alpha}-\sqrt{2} / 2 u_{\beta}=1
\end{aligned}
$$

\subsection{Matrizes de Decomposição}

Sem perda de generalidade, assume-se que o vetor de comando $\mathbf{u}_{c m d}$ está no tetraedro 1 , e que a seqüência de comutação implementada é $\mathbf{v}^{0}, \mathbf{v}^{8}, \mathbf{v}^{12}, \mathbf{v}^{14}, \mathbf{v}^{15}, \mathbf{v}^{14}, \mathbf{v}^{12}, \mathbf{v}^{8}$, $\mathbf{v}^{0}$. As durações em um período de comutação associados aos vetores não nulos $\mathbf{v}^{8}, \mathbf{v}^{12}$ e $\mathbf{v}^{14}$ são $\Delta t_{1}, \Delta t_{2}$ e $\Delta t_{3}$, respectivamente. Então, para que a tensão média produzida pelo inversor em um período de comutação seja igual a $\mathbf{u}_{c m d}$, a seguinte equação deve ser satisfeita:

$$
\mathbf{v}^{8} \Delta t_{1}+\mathbf{v}^{12} \Delta t_{2}+\mathbf{v}^{14} \Delta t_{3}=\mathbf{u}_{c m d} T_{s},
$$

onde: $\Delta t_{1}+\Delta t_{2}+\Delta t_{3}+\Delta t_{0}=T_{s}$.

Ainda, (18) pode ser reescrita na forma matricial:

$$
\left[\begin{array}{lll}
\mathbf{v}^{8} & \mathbf{v}^{12} & \mathbf{v}^{14}
\end{array}\right]\left[\begin{array}{lll}
\Delta t_{1} & \Delta t_{2} & \Delta t_{3}
\end{array}\right]^{\mathrm{T}}=\mathbf{u}_{c m d} T_{s} .
$$

Como os vetores que definem os vértices de cada tetraedro são linearmente independentes, os intervalos de tempo podem ser unicamente determinados por:

$$
\left[\begin{array}{lll}
\Delta t_{1} & \Delta t_{2} & \Delta t_{3}
\end{array}\right]^{\mathrm{T}}=\mathbf{M}_{1}\left[\begin{array}{lll}
u_{0} & u_{\alpha} & u_{\beta}
\end{array}\right]^{\mathrm{T}} T_{s}
$$

onde $u_{0}, u_{\alpha}$ e $u_{\beta}$ são as componentes do vetor de comando $\mathbf{u}_{c m d}$, e $\mathbf{M}_{1}=\left[\begin{array}{lll}\mathbf{v}^{8} & \mathbf{v}^{12} & \mathbf{v}^{14}\end{array}\right]^{-1}$ é a matriz de decomposição associada ao tetraedro 1. A duração dos vetores nulos são calculados da seguinte forma: $\Delta t_{0}=T_{s}-\Delta t_{1}-\Delta t_{2}-\Delta t_{3}$.

\subsection{Seqüências de Comutação}

Com relação à seqüência de vetores a ser implementada, levando em conta as considerações efetuadas nas seções anteriores, a Tabela 10 apresenta uma seqüência de comutação simétrica, onde os quatro braços do inversor comutam em alta freqüência, resultando em uma baixa THD da tensão de saída.

Tabela 10. Seqüência de comutação simétrica para o inversor trifásico com quatro braços a quatro fios.

Tetraedro $1 \quad \mathbf{v}^{0}-\mathbf{v}^{8}-\mathbf{v}^{12}-\mathbf{v}^{14}-\mathbf{v}^{15}-\mathbf{v}^{14}-\mathbf{v}^{12}-\mathbf{v}^{8}-\mathbf{v}^{0}$

Tetraedro $2 \quad \mathbf{v}^{0}-\mathbf{v}^{8}-\mathbf{v}^{12}-\mathbf{v}^{13}-\mathbf{v}^{15}-\mathbf{v}^{13}-\mathbf{v}^{12}-\mathbf{v}^{8}-\mathbf{v}^{0}$

Tetraedro $3 \quad \mathbf{v}^{0}-\mathbf{v}^{8}-\mathbf{v}^{9}-\mathbf{v}^{13}-\mathbf{v}^{15}-\mathbf{v}^{13}-\mathbf{v}^{9}-\mathbf{v}^{8}-\mathbf{v}^{0}$

Tetraedro $4 \quad \mathbf{v}^{0}-\mathbf{v}^{1}-\mathbf{v}^{9}-\mathbf{v}^{13}-\mathbf{v}^{15}-\mathbf{v}^{13}-\mathbf{v}^{9}-\mathbf{v}^{1}-\mathbf{v}^{0}$

Tetraedro $5 \quad \mathbf{v}^{0}-\mathbf{v}^{4}-\mathbf{v}^{12}-\mathbf{v}^{14}-\mathbf{v}^{15}-\mathbf{v}^{14}-\mathbf{v}^{12}-\mathbf{v}^{4}-\mathbf{v}^{0}$

Tetraedro $6 \quad \mathbf{v}^{0}-\mathbf{v}^{4}-\mathbf{v}^{12}-\mathbf{v}^{13}-\mathbf{v}^{15}-\mathbf{v}^{13}-\mathbf{v}^{12}-\mathbf{v}^{4}-\mathbf{v}^{0}$

Tetraedro $7 \quad \mathbf{v}^{0}-\mathbf{v}^{4}-\mathbf{v}^{5}-\mathbf{v}^{13}-\mathbf{v}^{15}-\mathbf{v}^{13}-\mathbf{v}^{5}-\mathbf{v}^{4}-\mathbf{v}^{0}$

Tetraedro $8 \quad \mathbf{v}^{0}-\mathbf{v}^{1}-\mathbf{v}^{5}-\mathbf{v}^{13}-\mathbf{v}^{15}-\mathbf{v}^{13}-\mathbf{v}^{5}-\mathbf{v}^{1}-\mathbf{v}^{0}$

Tetraedro $9 \quad \mathbf{v}^{0}-\mathbf{v}^{4}-\mathbf{v}^{6}-\mathbf{v}^{14}-\mathbf{v}^{15}-\mathbf{v}^{14}-\mathbf{v}^{6}-\mathbf{v}^{4}-\mathbf{v}^{0}$

Tetraedro $10 \quad \mathbf{v}^{0}-\mathbf{v}^{4}-\mathbf{v}^{6}-\mathbf{v}^{7}-\mathbf{v}^{15}-\mathbf{v}^{7}-\mathbf{v}^{6}-\mathbf{v}^{4}-\mathbf{v}^{0}$

Tetraedro $11 \quad \mathbf{v}^{0}-\mathbf{v}^{4}-\mathbf{v}^{5}-\mathbf{v}^{7}-\mathbf{v}^{15}-\mathbf{v}^{7}-\mathbf{v}^{5}-\mathbf{v}^{4}-\mathbf{v}^{0}$

Tetraedro $12 \quad \mathbf{v}^{0}-\mathbf{v}^{1}-\mathbf{v}^{5}-\mathbf{v}^{7}-\mathbf{v}^{15}-\mathbf{v}^{7}-\mathbf{v}^{5}-\mathbf{v}^{1}-\mathbf{v}^{0}$ 


\begin{tabular}{ll} 
Tetraedro 13 & $\mathbf{v}^{0}-\mathbf{v}^{2}-\mathbf{v}^{6}-\mathbf{v}^{14}-\mathbf{v}^{15}-\mathbf{v}^{14}-\mathbf{v}^{6}-\mathbf{v}^{2}-\mathbf{v}^{0}$ \\
Tetraedro 14 & $\mathbf{v}^{0}-\mathbf{v}^{2}-\mathbf{v}^{6}-\mathbf{v}^{7}-\mathbf{v}^{15}-\mathbf{v}^{7}-\mathbf{v}^{6}-\mathbf{v}^{2}-\mathbf{v}^{0}$ \\
Tetraedro 15 & $\mathbf{v}^{0}-\mathbf{v}^{2}-\mathbf{v}^{3}-\mathbf{v}^{7}-\mathbf{v}^{15}-\mathbf{v}^{7}-\mathbf{v}^{3}-\mathbf{v}^{2}-\mathbf{v}^{0}$ \\
Tetraedro 16 & $\mathbf{v}^{0}-\mathbf{v}^{1}-\mathbf{v}^{3}-\mathbf{v}^{7}-\mathbf{v}^{15}-\mathbf{v}^{7}-\mathbf{v}^{3}-\mathbf{v}^{1}-\mathbf{v}^{0}$ \\
Tetraedro 17 & $\mathbf{v}^{0}-\mathbf{v}^{2}-\mathbf{v}^{10}-\mathbf{v}^{14}-\mathbf{v}^{15}-\mathbf{v}^{14}-\mathbf{v}^{10}-\mathbf{v}^{2}-\mathbf{v}^{0}$ \\
Tetraedro 18 & $\mathbf{v}^{0}-\mathbf{v}^{2}-\mathbf{v}^{10}-\mathbf{v}^{11}-\mathbf{v}^{15}-\mathbf{v}^{11}-\mathbf{v}^{10}-\mathbf{v}^{2}-\mathbf{v}^{0}$ \\
Tetraedro 19 & $\mathbf{v}^{0}-\mathbf{v}^{2}-\mathbf{v}^{3}-\mathbf{v}^{11}-\mathbf{v}^{15}-\mathbf{v}^{11}-\mathbf{v}^{3}-\mathbf{v}^{2}-\mathbf{v}^{0}$ \\
Tetraedro 20 & $\mathbf{v}^{0}-\mathbf{v}^{1}-\mathbf{v}^{3}-\mathbf{v}^{11}-\mathbf{v}^{15}-\mathbf{v}^{11}-\mathbf{v}^{3}-\mathbf{v}^{1}-\mathbf{v}^{0}$ \\
Tetraedro 21 & $\mathbf{v}^{0}-\mathbf{v}^{8}-\mathbf{v}^{10}-\mathbf{v}^{14}-\mathbf{v}^{15}-\mathbf{v}^{14}-\mathbf{v}^{10}-\mathbf{v}^{8}-\mathbf{v}^{0}$ \\
Tetraedro 22 & $\mathbf{v}^{0}-\mathbf{v}^{8}-\mathbf{v}^{10}-\mathbf{v}^{11}-\mathbf{v}^{15}-\mathbf{v}^{11}-\mathbf{v}^{10}-\mathbf{v}^{8}-\mathbf{v}^{0}$ \\
Tetraedro 23 & $\mathbf{v}^{0}-\mathbf{v}^{8}-\mathbf{v}^{9}-\mathbf{v}^{11}-\mathbf{v}^{15}-\mathbf{v}^{11}-\mathbf{v}^{9}-\mathbf{v}^{8}-\mathbf{v}^{0}$ \\
Tetraedro 24 & $\mathbf{v}^{0}-\mathbf{v}^{1}-\mathbf{v}^{9}-\mathbf{v}^{11}-\mathbf{v}^{15}-\mathbf{v}^{11}-\mathbf{v}^{9}-\mathbf{v}^{1}-\mathbf{v}^{0}$ \\
\hline
\end{tabular}

\section{INVERSOR TRIFÁSICO TRÊS NÍVEIS A TRÊS FIOS}

Nesta seção é realizada uma análise do inversor trifásico três níveis mostrado na Figura 10. Cada braço do inversor possui 3 possíveis estados de condução $(\mathrm{P}, \mathrm{O}$ e $\mathrm{N})$ como mostrado na Tabela 11. Logo, os três braços do inversor produzem um total de 27 possíveis estados de condução (Seo et al., 2001).

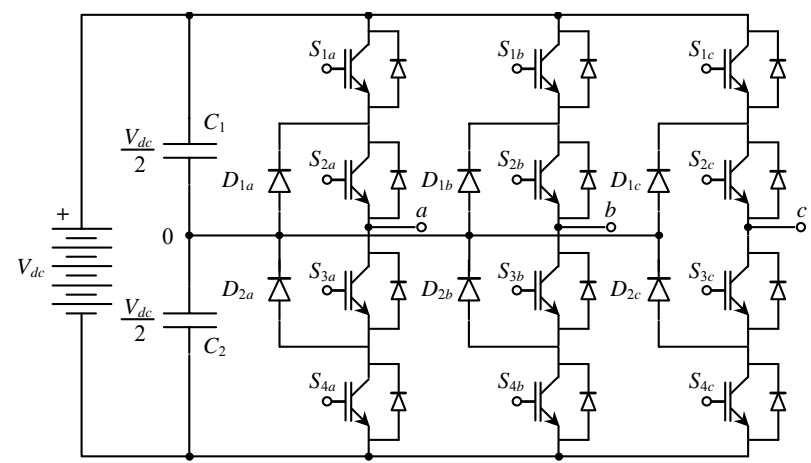

Figura 10. Inversor trifásico três níveis a três fios.

Tabela 11. Possíveis estados de condução e tensões de fase. $(x=a, b, c)$.

\begin{tabular}{cccccc}
\hline \multirow{2}{*}{ Símbolo } & \multicolumn{5}{c}{ Estados de comutação } \\
& $S_{1 x}$ & $S_{2 x}$ & $S_{3 x}$ & $S_{4 x}$ & Tensão de saída \\
\hline $\mathrm{P}$ & 1 & 1 & 0 & 0 & $V_{d c} / 2$ \\
$\mathrm{O}$ & 0 & 1 & 1 & 0 & 0 \\
$\mathrm{~N}$ & 0 & 0 & 1 & 1 & $-V_{d c} / 2$ \\
\hline
\end{tabular}

De forma similar ao inversor da seção 4 , as tensões de saída do inversor podem ser representadas em um espaço bidimensional. Então, utilizando (6), pode-se obter os vetores de comutação no espaço $\alpha \beta$ como apresentados na Tabela 12 e mostrados na Figura 11. Esses vetores foram normalizados em função da tensão sobre um capacitor do barramento $\mathrm{CC}$, isto é, $V_{d c} / 2$.
A partir da Tabela 12 e da Figura 11 é possível verificar que este inversor gera dezenove diferentes vetores de comutação. Note que alguns vetores de comutação podem ser gerados por dois ou três estados de condução diferentes, fornecendo um grau de liberdade adicional que pode ser utilizado, por exemplo, para controlar a tensão do ponto central do barramento CC. Os vetores de comutação podem ser agrupados em quatro diferentes classes de acordo com a sua amplitude (Seixas et al., 2000), ou seja: (Z) vetor zero $\left(\mathbf{v}^{0}\right)$ obtido a partir de três diferentes estados de condução; (S) vetores de pequena amplitude $\left(\mathbf{v}^{1}, \mathbf{v}^{2}, \mathbf{v}^{3}, \mathbf{v}^{4}, \mathbf{v}^{5}\right.$ e $\left.\mathbf{v}^{6}\right)$ que podem ser obtidos a partir de dois diferentes estados de condução; (M) vetores de média amplitude $\left(\mathbf{v}^{8}, \mathbf{v}^{10}, \mathbf{v}^{12}, \mathbf{v}^{14}\right.$, $\mathbf{v}^{16}$ e $\mathbf{v}^{18}$ ) que podem ser obtidos a partir de somente um estado de condução e (L) vetores de grande amplitude $\left(\mathbf{v}^{7}\right.$, $\mathbf{v}^{9}, \mathbf{v}^{11}, \mathbf{v}^{13}, \mathbf{v}^{15}$ e $\left.\mathbf{v}^{17}\right)$ que também só podem ser obtidos a partir de somente um estado de condução.

Tabela 12. Possíveis vetores de comutação no espaço $\alpha \beta$ para o inversor trifásico três níveis

\begin{tabular}{ccc}
\hline Estados de comutação & {$\left[\begin{array}{ll}\alpha & \beta\end{array}\right]^{\mathrm{T}}$} & Vetor \\
\hline NNN - OOO -PPP & {$\left[\begin{array}{ll}0 & 0\end{array}\right]$} & $\mathbf{v}^{0}$ \\
POO - ONN & {$\left[\begin{array}{ll}\sqrt{6} / 3 & 0\end{array}\right]$} & $\mathbf{v}^{1}$ \\
PPO - OON & {$\left[\begin{array}{ll}\sqrt{6} / 6 & \sqrt{2} / 2\end{array}\right]$} & $\mathbf{v}^{2}$ \\
OPO - NON & {$\left[\begin{array}{ll}-\sqrt{6} / 6 & \sqrt{2} / 2\end{array}\right]$} & $\mathbf{v}^{3}$ \\
OPP - NOO & {$\left[\begin{array}{ll}-\sqrt{6} / 3 & 0\end{array}\right]$} & $\mathbf{v}^{4}$ \\
OOP - NNO & {$\left[\begin{array}{ll}-\sqrt{6} / 6 & -\sqrt{2} / 2\end{array}\right]$} & $\mathbf{v}^{5}$ \\
POP - ONO & {$\left[\begin{array}{ll}\sqrt{6} / 6 & -\sqrt{2} / 2\end{array}\right]$} & $\mathbf{v}^{6}$ \\
PNN & {$\left[\begin{array}{ll}2 \sqrt{6} / 3 & 0\end{array}\right]$} & $\mathbf{v}^{7}$ \\
\hline
\end{tabular}

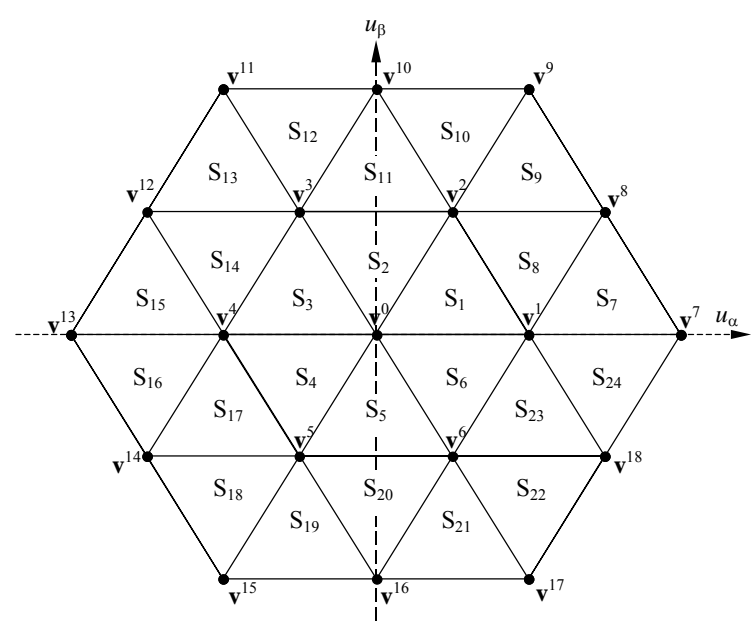

Figura 11. Espaço das tensões de saída do inversor trifásico três níveis em coordenadas $\alpha \beta$. 


\begin{tabular}{|c|c|c|}
\hline PON & {$\left[\begin{array}{ll}\sqrt{6} / 2 & \sqrt{2} / 2\end{array}\right]$} & $\mathbf{v}^{8}$ \\
\hline PPN & {$\left[\begin{array}{ll}\sqrt{6} / 3 & \sqrt{2}\end{array}\right]$} & $\mathbf{v}^{9}$ \\
\hline OPN & {$\left[\begin{array}{ll}0 & \sqrt{2}\end{array}\right]$} & $\mathbf{v}^{10}$ \\
\hline NPN & {$\left[\begin{array}{ll}-\sqrt{6} / 3 & \sqrt{2}\end{array}\right]$} & $\mathbf{v}^{11}$ \\
\hline NPO & {$\left[\begin{array}{ll}-\sqrt{6} / 2 & \sqrt{2} / 2\end{array}\right]$} & $\mathbf{v}^{12}$ \\
\hline NPP & {$\left[\begin{array}{ll}-2 \sqrt{6} / 3 & 0\end{array}\right]$} & $\mathbf{v}^{13}$ \\
\hline NOP & {$\left[\begin{array}{ll}-\sqrt{6} / 2 & -\sqrt{2} / 2\end{array}\right]$} & $\mathbf{v}^{14}$ \\
\hline NNP & {$[-\sqrt{6} / 3 \quad-\sqrt{2}]$} & $\mathbf{v}^{15}$ \\
\hline ONP & {$\left[\begin{array}{ll}0 & -\sqrt{2}\end{array}\right]$} & $\mathbf{v}^{16}$ \\
\hline PNP & {$\left[\begin{array}{ll}\sqrt{6} / 3 & -\sqrt{2}\end{array}\right]$} & $\mathbf{v}^{17}$ \\
\hline PNO & {$\left[\begin{array}{ll}\sqrt{6} / 2 & -\sqrt{2} / 2\end{array}\right]$} & $\mathbf{v}^{18}$ \\
\hline
\end{tabular}

\subsection{Planos de Separação e Planos Limites}

Na Figura 12 estão identificados os planos de separação dos setores e os planos limites do espaço das tensões de saída para a operação na região linear.

As equações que descrevem cada um dos planos de separação em coordenadas $\alpha \beta$ podem ser dadas por:

$$
\begin{gathered}
\mathrm{PS}_{1} \therefore u_{\beta}-\sqrt{3} u_{\alpha}-\sqrt{2}=0 \quad \mathrm{PS}_{4} \therefore u_{\beta}+\sqrt{3} u_{\alpha}-\sqrt{2}=0 \\
\mathrm{PS}_{2} \therefore u_{\beta}-\sqrt{3} u_{\alpha}=0 \quad \mathrm{PS}_{5} \therefore u_{\beta}+\sqrt{3} u_{\alpha}=0 \\
\mathrm{PS}_{3} \therefore u_{\beta}-\sqrt{3} u_{\alpha}+\sqrt{2}=0 \quad \mathrm{PS}_{6} \therefore u_{\beta}+\sqrt{3} u_{\alpha}+\sqrt{2}=0 \\
\mathrm{PS}_{7} \therefore u_{\beta}-\frac{\sqrt{2}}{2}=0 \quad \mathrm{PS}_{8} \therefore u_{\beta}=0 \quad \mathrm{PS}_{9} \therefore u_{\beta}+\frac{\sqrt{2}}{2}=0
\end{gathered}
$$

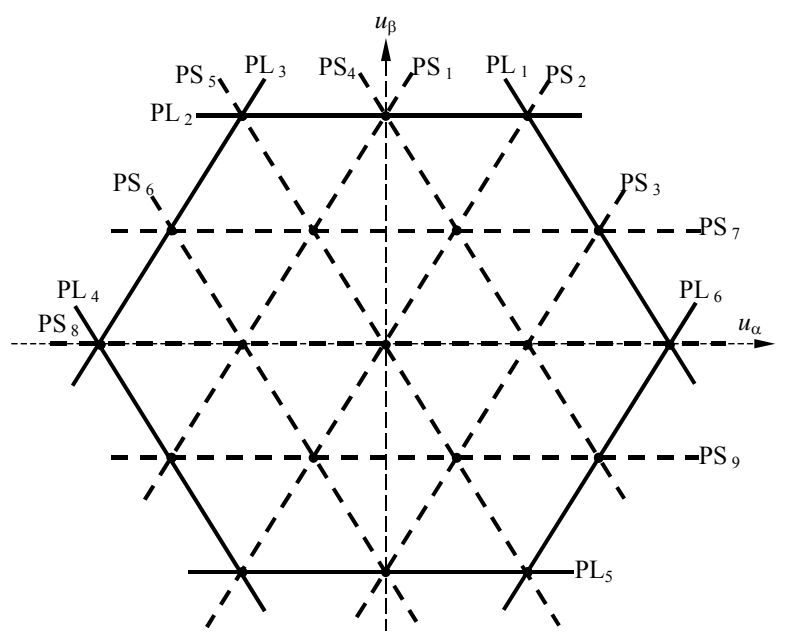

Figura 12. Planos limites e de separação dos setores no espaço $\alpha \beta$ para o inversor trifásico três níveis.

As equações dos planos limites podem ser representadas por:

$$
\begin{array}{ll}
\mathrm{PL}_{1} \therefore u_{\beta}+\sqrt{3} u_{\alpha}-2 \sqrt{2}=0 & \mathrm{PL}_{4} \therefore u_{\beta}+\sqrt{3} u_{\alpha}+2 \sqrt{2}=0 \\
\mathrm{PL}_{2} \therefore u_{\beta}-\sqrt{2}=0 & \mathrm{PL}_{5} \therefore u_{\beta}+\sqrt{2}=0 \\
\mathrm{PL}_{3} \therefore u_{\beta}-\sqrt{3} u_{\alpha}-2 \sqrt{2}=0 & \mathrm{PL}_{6} \therefore u_{\beta}=\sqrt{3} u_{\alpha}-2 \sqrt{2}
\end{array}
$$

\subsection{Matrizes de Decomposição}

Sem perda de generalidade, assume-se que o vetor de comando $\mathbf{u}_{c m d}$ está no Setor 1 . Os vetores de comutação utilizados são $\mathbf{v}^{0}, \mathbf{v}^{1}$ e $\mathbf{v}^{2}$, e as correspondentes durações de cada vetor são $\Delta t_{0}, \Delta t_{1}$, e $\Delta t_{2}$, respectivamente. Assim, de forma semelhante às seções anteriores tem-se que:

$$
\mathbf{v}^{0} \Delta t_{0}+\mathbf{v}^{1} \Delta t_{1}+\mathbf{v}^{2} \Delta t_{2}=\mathbf{u}_{c m d} T_{s}
$$

onde: $\Delta t_{0}+\Delta t_{1}+\Delta t_{2}=T_{s}$.

Assim, rescrevendo a equação (21) na forma matricial, é possível calcular as durações dos vetores de comutação em $T_{s}$ :

$$
\left[\begin{array}{c}
\Delta t_{0} \\
\Delta t_{1} \\
\Delta t_{2}
\end{array}\right]=\mathbf{M}_{1}\left[\begin{array}{c}
u_{\alpha} \\
u_{\beta} \\
1
\end{array}\right] T_{s} \therefore \mathbf{M}_{1}=\left[\begin{array}{ccc}
\mathbf{v}^{0} & \mathbf{v}^{1} & \mathbf{v}^{2} \\
1 & 1 & 1
\end{array}\right]^{-1}
$$

onde $u_{\alpha}$ e $u_{\beta}$ são as componentes do vetor de comando e $\mathbf{M}_{1}$ é a matriz de decomposição associada, neste caso, ao Setor 1.

Finalizando, uma transformação linear que possibilita reduzir o esforço computacional associado a identificação dos setores e ao cálculo das durações dos vetores de comutação para inversores multiníveis é apresentado por Celanovic et al, 2001.

\subsection{Seqüências de Comutação}

De forma semelhante às outras topologias contidas neste artigo, a escolha da seqüência de comutação para um determinado setor deve minimizar o número de comutações dos interruptores, bem como reduzir a THD nas tensões de saída.

No caso do inversor três níveis, ainda existe o problema do desbalanceamento das tensões dos capacitores que compõem o barramento CC. Como existem vetores de comutação que afetam de forma distinta a tensão do ponto neutro, uma possível solução é mudar a seqüência de comutação de tal forma que os capacitores sejam carregados ou descarregados (McGrath et al., 2001; Rojas et al., 1995; Seo et al., 2001). Uma outra solução, consiste 
em distribuir de forma adequada os vetores de comutação redundantes (Wei et al., 1999). Ambas estratégias são baseadas em função do nível de tensão do ponto neutro e do sentido da corrente nos capacitores.

\section{RESULTADOS EXPERIMENTAIS}

Para validar a metodologia proposta nas seções anteriores, resultados experimentais de duas das cinco topologias analizadas são apresentados. Estes resultados foram obtidos com a utilização de um inversor trifásico com quatro braços e um filtro LC $(L=250 \mu \mathrm{H}, C=60 \mu \mathrm{F})$ de saída. Um DSP de ponto fixo TMS320F241 foi utilizado para a implementação dos algoritmos de modulação.

A Figura 13 apresenta resultados experimentais relacionados à modulação space vector do inversor de tensão com três braços a três fios. A Figura 13(a) mostra o sinal de comando das chaves e a Figura 13(b) as tensões de linha, $v_{a b}$ e $v_{b c}$ sintetizadas por esse inversor. De forma similar, a Figura 14(a) apresenta os sinais de comando das chaves e a Figura 14(b) mostra as tensões de fase sintetizadas pelo inversor com quatro braços a quatro fios.

Finalmente, a Figura 15(a) mostra as tensões de fase, e a Figura 15(b) apresenta as tensões de linha, logo após o filtro LC, quando o vetor de comando $\mathbf{u}_{c m d}$ é limitado por um algoritmo de limitação que utiliza as equações dos planos limites apresentados em (17). Embora as tensões de fase apresentem algumas componentes harmônicas de baixa ordem, com a utilização deste algoritmo é possível explorar toda a capacidade de geração de tensão, do inversor com quatro braços a quatro fios.

\section{CONCLUSÕES}

Este artigo demonstra que a técnica de modulação do tipo space vector aplicada a inversores alimentados em tensão pode ser tratada de uma forma unificada. O procedimento para a implementação digital desta modulação identifica cinco etapas básicas. Estas etapas foram aplicadas, neste caso, a cinco topologias de inversores de tensão freqüentemente encontradas na literatura, que permitiram exemplificar a metodologia proposta. Para cada um dos inversores foram apresentados os possíveis vetores de comutação, os planos de separação, os planos limites que definem a operação do inversor na região linear, bem como algumas possíveis seqüências de comutação que podem ser implementadas. Finalmente, apresentaram-se resultados experimentais de dois inversores de tensão trifásicos, a três braços três fios e a quatro braços quatro fios, para validar a metodologia proposta.

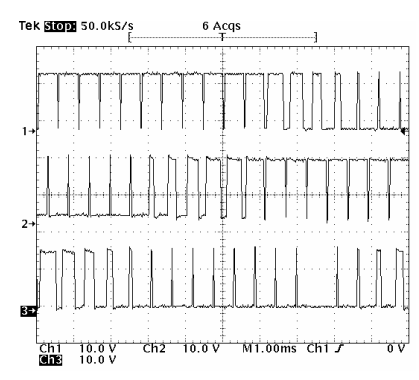

(a)

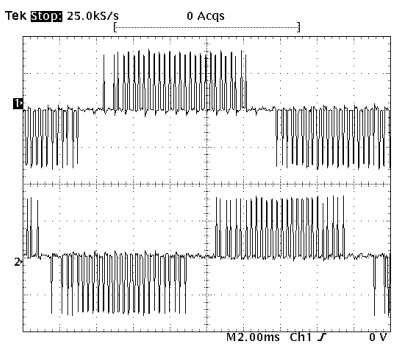

(b)
Figura 13 - Resultados experimentais. Inversor trifásico três braços a três fios. (a) Padrão PWM das chaves. (b) Tensões PWM de linha, $v_{a b}$ e $v_{b c}$.

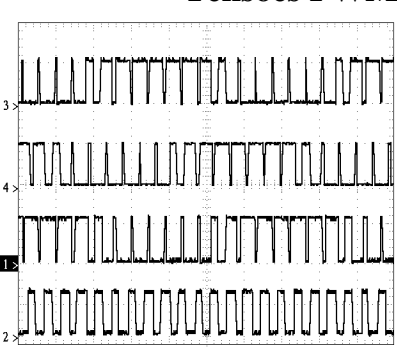

(a) (b)

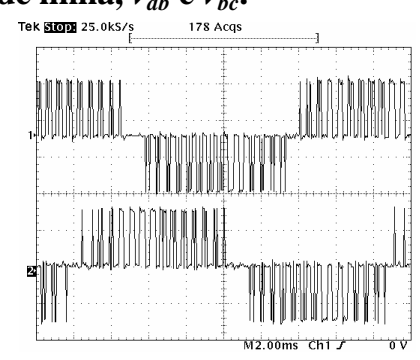

Figura 14 - Resultados experimentais. Inversor trifásico quatro braços a quatro fios. (a) Padrão PWM das chaves. (b) Tensões PWM de fase, $v_{a}$ e $v_{b}$.

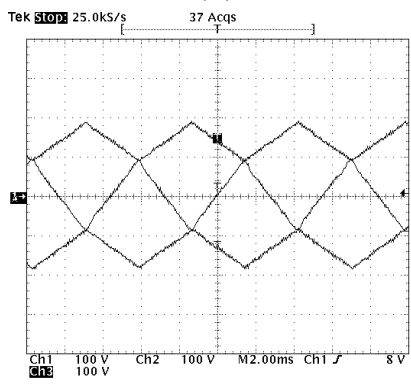

(a)

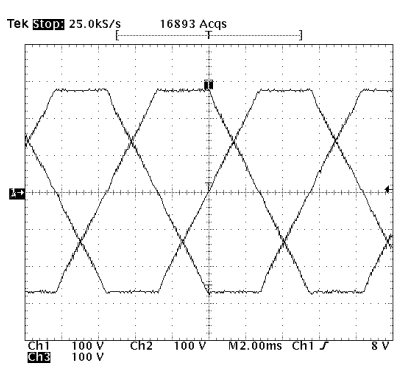

(b)
Figura 15 - Resultados experimentais. Vetor de comando $\mathbf{u}_{c m d}$ limitado pelos planos limites. (a) Tensões de fase. (b) Tensões de linha. Escala vertical: $100 \mathrm{~V} /$ div. Escala horizontal: $2 \mathrm{~ms} / \mathrm{div}$.

\section{REFERÊNCIAS BIBLIOGRÁFICAS}

Bakhshai, A. R., Rad, H. R. S., Joos, G. (2001) Space vector modulation based on classification method in three-phase multi-level voltage source inverters. IEEE IAS Annual Meet.: 597-602.

Boost, M. A., Ziogas, P. D. (1988). State-of-the-art carrier techniques: A critical evaluation. IEEE Trans. Ind. Applicat., 24(2): 271-279.

Botterón, F., Pinheiro, H., Gründling, H. A., Pinheiro, J. R., Hey, H. L. (2001) Digital voltage and current 
controllers for three-phase PWM inverter for UPS applications. IEEE IAS Annual Meeting: CD-ROM.

Bowes, S. R., Lai, Y. S. (1997). The relationship between space-vector modulation and regular-sampled PWM. IEEE Trans. Ind. Electr., 44(5): 670-679.

Camargo, R. F., Botterón, F., Pinheiro, H., Gründling, H. A., Pinheiro, J. R., Hey, H. L. (2002) Discrete control of three-phase four-leg PWM inverter. IEEE PESC Conf. Rec.: CD-ROM.

Casadei, D., Serra, G., Tani, A., Zarri, L. (2002). Matrix converter modulation strategies: A new general approach based on space-vector representation of the switch state. IEEE Trans. Ind. Electr., 49(2): 370-381.

Celanovic, N., Boroyevich, D. (2001). A fast space-vector modulation algorithm for multilevel three-phase converters. IEEE Trans. Ind. Applicat., 37(2): 637-641.

Doval-Gandoy, J., Iglesias, A., Castro, C., Peñalver, C. M. (1999). Three alternatives for implementing space vector modulation with the DSP TMS320F240. IEEE IECON Proc.: 336-341.

Enjeti, P. N., Ziogas, P. D., Lindsay, J. F. (1990). Programmed PWM techniques to eliminate harmonics: A critical evaluation. IEEE Trans. Ind. Applicat., 26(2): 302-316.

Holtz, J. (1992). Pulsewidth modultation - A survey. IEEE Trans. Ind. Electr., 39(5): 410-419.

Huber, L., Boroyevich, D. (1995). Space vector modulated three-phase to three-phase matrix converter with input power factor correction. IEEE Trans. Ind. Applicat., 31(6): 1234-1246.

Jenni, F., Wueest, D. (1993). The optimization parameters of space vector modulation. EPE Conf. Proc.: 376381.

McGrath, B. P., Holmes, D. G., Lipo, T. A. (2001). Optimized space vector switching sequences for multilevel inverters. IEEE PESC Conf. Rec.: 11231129.

Patel, H. S., Hoft, R. G. (1973). Generalized techniques of harmonic elimination and voltage control in thyristor inverters: Part I - Harmonic Elimination. IEEE Trans. Ind. Applicat., 9(3): 110-117.

Pinheiro, H., Blume, R., Jain, P. (2000). Space vector modulation method for single-phase on-line threeleg UPS. INTELEC Conf. Proc.: 679-686.

Rahman, M. A., Quaicoe, J. E., Choudhury, M. A. (1987). Performance analysis of delta modulated PWM inverters. IEEE Trans. Power Electr., 2(3): 227-232.

Ranganathan, V. T. (1997). Space vector pulsewidth modulation - A status review. Sãdhanã, 22(6): pp. 675-688.

Rojas, R., Ohnishi, T., Suzuki, T. (1995) An improved voltage vector control method for neutral-pointclamped inverters. IEEE Trans. Power Electr., 10(6): 666-672.

Ryan, M. J., De Doncker, R. W., Lorenz, R. D. (1999) Decoupled control of a four-leg inverter via a new $4 \times 4$ transformation matrix. IEEE PESC Conf. Rec.: 187-192.

Ryan, M. J., Lorenz, R. D., De Doncker, R. W. (1999) Modeling of multileg sine-wave inverters: A geometric approach. IEEE Trans. Ind. Electr., 46(6): 1183-1191.

Seixas, P. F., Mendes, M. A. S., Donoso-Garcia, P., Lima, A. M. N. (2000) A space vector PWM method for three-level voltage source inverters. IEEE PESC Conf. Rec.: 549-555.

Seo, J. H., Choi, C. H., Hyun, D. S. (2001). A new simplified space-vector PWM method for three-level inverters. IEEE Trans. Power Electr., 16(4): $545-550$.

Van Der Broeck, H. W., Skudelny, H. C., Stanke, G. V. (1988). Analysis and realization of a pulsewidth modulator based on voltage space vectors. IEEE Trans. Ind. Applicat., 24(1): 142-150.

Wang, F. (2000). Sine-triangle vs. space vector modulation for three-level PWM voltage source inverters. IAS Annual Meet.: 2482-2488.

Wei L., Wu L., Li C., Wang H., Li S. (1999). A novel space vector control of three-level PWM converter. IEEE PEDS Conference: 745-750.

Zhan, C., Arulampalam, A., Ramachandaramurthy, V. K., Fitzer, C., Barnes, M., Jenkins, N. (2001). Novel voltage space vector PWM algorithm of 3-phase 4wire power conditioner. IEEE Power Eng. Soc. Winter Meet.: 1045-1050.

Zhang, R., Boroyevich, D., Prasad, V. H. (1997) A threephase inverter with a neutral leg with space vector modulation. IEEE APEC Proc., (2): 864-870.

Ziogas, P. D. (1981). The delta modulation techniques in static PWM inverters. IEEE Trans. Ind. Applicat., 17(2): 199-204. 